\title{
Protée
}

\section{Intertextualité et déconstruction}

\section{Eli Rozik}

Volume 27, numéro 1, 1999

La Mort de Molière et des autres

URI : https://id.erudit.org/iderudit/030551ar

DOI : https://doi.org/10.7202/030551ar

Aller au sommaire du numéro

Éditeur(s)

Département des arts et lettres - Université du Québec à Chicoutimi

ISSN

0300-3523 (imprimé)

1708-2307 (numérique)

Découvrir la revue

Citer cet article

Rozik, E. (1999). Intertextualité et déconstruction. Protée, 27(1), 111-116.

https://doi.org/10.7202/030551ar

\section{Résumé de l'article}

Une série de séquences qui servent à suggérer la mort de Molière constitue l'architecture de cette vidéo. Apparemment, ces plans servent à créer une image englobante de la mort de l'illustre dramaturge - le dernier chapitre de son bio-texte - tel qu'on peut le connaître par les biographies et les livres d'histoire. Mais ces images et ces citations sont en fait des métonymies d'un immense corpus de textes qui, depuis des siècles, remplissent cette fonction cruciale de créer l'image d'un héros culturel, d'un champion de la critique sociale. Cependant, cette interprétation apparemment historique de sa mort est minée par une série d'intertextes, qui viennent jusqu'à inverser l'image établie de Molière. Ces intertextes incluent ses propres comédies, dont Le Malade imaginaire, Dom Juan et Le Tartuffe. 


\section{INTERTEXTUALITÉ ET DÉCONSTRUCTION}

Traduit de l'anglais par Jean-Pierre Vidal

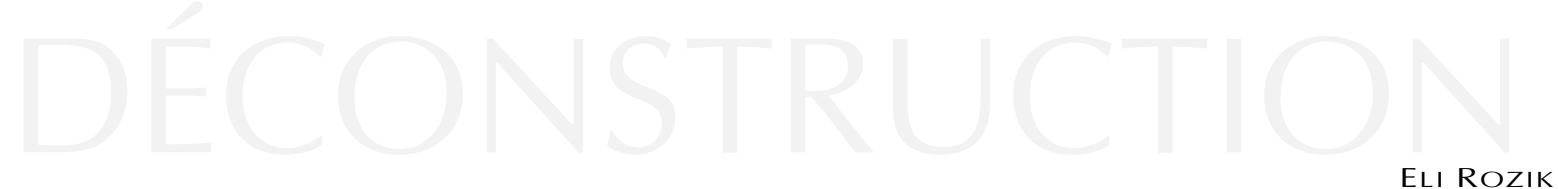

L'architecture de La Mort de Molière ${ }^{1}$ repose sur plusieurs séquences visant à convoquer une image de Molière mourant. Mais loin de créer une image d'ensemble de l'illustre dramaturge sur ses derniers jours, ces séquences au contraire la défont sans cesse. Le dernier chapitre de la vie de Molière, de son biotexte - telle que nous la révèle l'immense corps de biographies et de livres d'histoire qui depuis des siècles ont répandu l'image d'un critique des vices de la société - est ici travaillé d'une série de contrastes sous forme de relations intertextuelles qui œuvrent à déconstruire l'image bien établie que la culture nous a léguée.

Je me propose, dans cet article, de mettre l'accent sur le rôle crucial des relations intertextuelles entre la vidéo et les propres comédies de Molière, principalement Le Malade imaginaire, Dom Juan et Le Tartuffe. J'ai l'intention en particulier d'insister sur l'importance des indicateurs non verbaux qui montrent ces relations. Je travaillerai, en l'occurrence, à partir de la définition suivante, de ce que j'appelle un «indicateur non verbal»: toute "image» visuelle ou auditive qui rappelle un texte connu et introduit des éléments sémantiques, dénotatifs et/ou connotatifs, qui exercent une fonction bien précise dans la structure d'ensemble et la signification générale de l'univers fictif décrit par un texte.

\section{LE MALADE IMAGINAIRE}

L'image visuelle de Molière gisant dans son lit introduit en intertexte Le Malade imaginaire $^{2}$ dans la mesure où elle forme une réplique visuelle de l'image typique d'Argan dans la scène d'ouverture de nombre de mises en scène de cette comédie. Comme, d'après les indications scéniques du dramaturge lui-même, Argan devrait être plutôt assis dans un fauteuil, éventuellement celui qui est conservé sous verre dans le foyer de la Comédie-Française, l'image visuelle de cette célèbre relique présentée dans la vidéo devrait également être perçue comme un indicateur non verbal de cette relation intertextuelle. Ces images agissent ainsi de façon synonymique comme des allusions à la fois au Malade imaginaire et au bio-texte de Molière, en tant qu'auteur et acteur qui créa et joua le rôle de l'hypocondriaque. Comme il était lui-même vraiment malade et atteint à ce moment-là de la maladie qui devait l'emporter tandis qu'il jouait ce rôle, ces images rendent incertaine la frontière entre le texte artistique et le bio-texte. 
Grimarest nous a transmis l'anecdote de l'effondrement de Molière au cours de la troisième représentation de la pièce, où il fut saisi de convulsions en prononçant le mot «juro» (p.458) qui fait partie de la parodie de la cérémonie en latin au cours de laquelle les nouveaux médecins prononcent le serment d'Hippocrate. L'image visuelle des convulsions et de l'hémorragie subies par Molière forme ainsi un indicateur non verbal supplémentaire de cette relation intertextuelle. Il se peut que Molière ait tenu le rôle d'Argan parce qu'il éprouvait certaines affinités avec le personnage. Son effondrement sur scène, en jouant le rôle, et finalement la mort qui s'en est suivie, plaident en faveur de cette intuition. En jouant le rôle parodique d'un docteur, Toinette pourrait bien venir souligner le parallélisme entre la vie et la fiction lorsqu'elle déclare qu'Argan souffre d'une affection pulmonaire (III, $x$ ), diagnostic que confirment les crachements de sang ${ }^{3}$ réels de Molière.

On peut aussi attribuer cette analogie à l'intuition qu'aurait eue Molière d'avoir produit avec ce personnage d'hypocondriaque une projection et une métaphore comique de lui-même et qu'en jouant le rôle d'Argan il se désignait lui-même comme l'objet du procès métaphorique. Cette relation trouve son écho et même se renforce dans le fait que Wilson joue le rôle de Molière. De plus, en concevant le personnage d'Argan et en le jouant, Molière montre qu'il est parvenu à dissoudre dans l'humour ses propres angoisses quant à la maladie et la mort: «Molière c'est la naissance de la comédie venant de l'esprit / De dégoût» (section 1). Ce mécanisme devrait être envisagé comme la fonction la plus profonde et la plus importante de la comédie.

L'ironie s'exerce à l'endroit de Molière qui, ayant ri de la crainte de la mort, est lui-même vraiment en train de mourir. Dans le texte de Kafka cité à la section 4, quand l'enfant supplie qu'on le sauve, le docteur se plaint: «Ils exigent toujours l'impossible du médecin [...] le médecin doit tout faire de sa main légère de chirurgien ". Un tel commentaire contraste avec le sous-texte des comédies de Molière et reflète une humilité fort raisonnable ainsi qu'une conscience aiguë des limites de la médecine. Ironiquement, l'effondrement de Molière en scène, au moment même où il va prêter le serment qu'il tourne en dérision, devient une sorte de vengeance divine qui s'exerce sur l'homme qui s'est moqué à la fois des craintes infondées de l'hypocondriaque et des prétentions des médecins.

Le motif de la crainte de la maladie et de la mort introduit par l'hypocondriaque sous-tend plusieurs des textes cités dans l'œuvre de Wilson: l'attribution par Plutarque de la mort de Sylla à une prolifération de poux (section 2); Kafka décrivant la mort d'un enfant produite par d'énormes vers infestant sa peau (section 4); Mattaüs von Collin évoquant la mort d'une reine aux mains de son amant nain (section 6); et la description que fait Ernest Hemingway des souffrances des soldats italiens déchiquetés à mort par des vautours, en Éthiopie (section 10). Toutes ces histoires ont en commun d'illustrer le motif de «la vie humaine anéantie par des espèces inférieures", telles les poux, les vers et les vautours. Le nain introduit le thème de la beauté annihilée par la laideur et la difformité. La lettre d'Edwin Denby ajoute à cela le traitement ironique de la toux utilisée pour éloigner les démons et la mort (section 7). Tous ces textes viennent appuyer les mots de Müller - «Un lambeau de Shakespeare/Au paradis des bactéries" -, qui reflètent le paradoxe de l'annihilation du plus grand esprit (Shakespeare) par les formes de vie les plus basses (les bactéries).

Comme Shakespeare, Molière est lui aussi dévoré par des «bactéries» qui, comme les poux, les vers et les vautours, ne sont pas seulement des agents de la mort mais aussi des symboles de désintégration. De plus, dans un contexte de «bactéries», la métaphore du "paradis» (qui devrait être comprise comme une vie totalement immergée dans l'ordre de la nature, c'est-àdire privée de conscience humaine) crée un contraste elliptique avec l'accomplissement spirituel, qui devient ainsi une métonymie implicite de l'enfer.

Contrastant avec les autres personnages qui sont vêtus de costumes d'époque, le docteur porte une blouse blanche moderne. Cette exception à la règle des costumes rattache l'image visuelle du médecin en tant qu'indicateur intertextuel de tous les médecins qu'on retrouve dans les pièces de Molière - à la 
science moderne et en particulier à l'image de Galilée montrée dans la vidéo. Vue à travers le prisme de cette métonymie particulière, la science nous révèle qu'il reste matière à émerveillement dans l'énigme du génie telle que la vie (la métaphore visuelle de la comète) et la mort (causée par des bactéries) de Molière l'illustrent. Le docteur médite non seulement sur son effondrement mais aussi sur son fauteuil vide, renvoyant par là à l'incapacité existentielle de la science de comprendre l'accomplissement spirituel dans le contexte des lois de la nature.

\section{LE TARTUFFE}

L'image récurrente des religieuses qui s'occupent de Molière sur son lit de mort produit une relation intertextuelle avec Le Tartuffe. Ironiquement, le "critique moralisateur" qui avait dénoncé la bigoterie du clergé, lui-même maintenant assoiffé de réconfort divin, est assisté par un chœur de religieuses. Mais leur attitude manifeste d'indifférence à ses souffrances vient contredire la nature compatissante qu'on leur prête, encore qu'elle convienne à ce qu'on peut supposer être leur sentiment à l'endroit d'un hérétique. Cette attitude est semblable à celle du docteur qui, en fait, ne montre pas non plus la moindre intention d'aider Molière et ne manifeste que l'intérêt qu'il éprouve à tenter de comprendre le phénomène «Molière». De plus, cette indifférence est partagée par tous ceux qui entourent Molière sur son lit de mort. Dans son intérêt pour les étoiles, Galilée lui-même est indifférent au sort de l'humanité:

Les étoiles ne se soucient pas / Du hasard de l'humanité, [...] /

[...] Le résultat / Dépend d'autres circonstances

Que le cours des étoiles. (section 1)

Les sœurs se livrent à une pratique qui rappelle davantage un rite païen qu'une cérémonie chrétienne: placées de chaque côté du carré de plancher éclairé, elles se touchent les paumes, tapent dans leurs mains au dessus de leur tête et se frappent la poitrine de leurs poings en hurlant. Le rituel auquel elles se livrent autour du carré de plancher éclairé - qui représente probablement une scène - en fait plus des acteurs que des religieuses. Cette image visuelle est en relation avec celle du docteur, qui goûte l'urine de Molière et la fait rouler dans sa bouche comme s'il s'agissait d'un grand cru. Dans ses efforts pour comprendre le phénomène Molière, il adopte une sorte de comportement de sorcier, ce qui vient renforcer le mot de Müller: «La médecine est une des grandes erreurs de l'humanité» (section 4). C'est là une adaptation du mot de Dom Juan à Sganarelle quand celui-ci se déguise en médecin (III, 1). Les médecins restent un objet d'ironie et de rire malgré leur passage de la sorcellerie à la science. Ces images ironisent à la fois sur les médecins et leur prétention à la science véritable, et sur les religieuses et leur prétention d'être des servantes de Dieu au service de l'humanité. Elles viennent ainsi en renfort de la critique des faux-dévots et des faux-médecins à laquelle s'est livré Molière.

\section{DOM JUAN}

En écrivant Le Tartuffe, le but avoué de Molière était de s'attaquer à l'hypocrisie religieuse. La pièce eut à l'époque un impact considérable et Molière en ressortit comme le gardien zélé de la sincérité religieuse. L'interdiction de la pièce (12 mai 1664) l'amena à écrire et à monter rapidement Dom Juan (15 février 1665) ${ }^{4}$. L'intention initiale de Molière était de critiquer un «vice à la mode» $(\mathrm{V}, 2)$ non seulement pour sa recherche du plaisir sexuel sans entraves, mais aussi pour sa négation résolue de tout ce qui avait de la valeur pour la société de son temps. Ce personnage mythologique, outre sa totale hérésie, devient aussi dans les mains de Molière un «faux-dévot", c'est-à-dire un hypocrite qui a appris que se faire passer pour un dévot était le meilleur moyen de parvenir à ses fins égoïstes. Satan se déguise ainsi en ange: «Je m'érigerai en censeur des actions d'autrui, jugerai mal de tout le monde et n'aurai bonne opinion que de moi» $(\mathrm{V}, 2)$.

Molière lui-même a souvent été accusé d'adopter la même attitude. Montfleury l'accusa même d'avoir commis l'inceste en épousant sa propre fille, Armande $^{5}$. Si la chose est vraie, Molière avait ainsi commis un crime bien plus infâme que tous ceux, à vrai dire plutôt mythiques, attribués à Dom Juan. Müller cite Sganarelle qui décrit, entre autres choses, son maître comme «un hérétique, qui ne croit ni ciel, 
ni enfer [...] un pourceau d'Épicure, [...] qui [...] traite de billevesées tout ce que nous croyons" (I, 1 cf. section 3). Mais Dom Juan du moins ne commit jamais d'inceste. Apparemment, Wilson et Müller adoptent la version la moins compromettante pour Molière: «La mère de sa dernière femme/ Était sa première» (section 1); mais la suite: «La France est une seule famille» implique le pire: si l'inceste est la règle, il devient justifié de dire de Molière qu'il est «la puanteur de Versailles» (section 1).

L'image visuelle du scarabée, sortant de la bouche de Madeleine et tombant sur le dos de la main droite de Molière, sur l'annulaire, comme un sceau royal, rappelle le scarabée sacré de l'ancienne Égypte. Cette image lie le regard accusateur de Madeleine à la permission donnée aux anciens pharaons d'épouser leur propre sœur. Le scarabée porte ainsi un message non verbal ambigu, qui représente Molière à la fois comme une figure royale et comme un père coupable d'inceste.

Cet enchevêtrement familial explique que Madeleine, déjà morte depuis un an au moment de la mort de Molière, apparaisse ici comme une image visuelle qui rappelle intertextuellement deux personnages de la pièce: le spectre sous la forme d'une femme voilée $(\mathrm{V}, 5)$ et le commandeur mort sous la forme d'une statue $(\mathrm{V}, 6)$, les deux personnages qui conduisent Dom Juan à sa perte. Alors que le spectre est une figure allégorique qui fait une dernière tentative pour convaincre Dom Juan de se repentir, la statue, elle, exécute la punition de Dom Juan: sa descente dans la tombe et le feu de l'enfer:

Dom Juan: O Ciel! Que sens-je? Un feu invisible me brûle, je n'en puis plus, et tout mon corps devient un brasier ardent. Ah!

(Le tonnerre tombe avec un grand bruit et de grands éclairs sur Dom Juan. La terre s'ouvre et l'abîme; et il sort de grands feux de l'endroit où il est tombé.) $(\mathrm{V}, 6)$

Cette image visuelle, typique des représentations traditionnelles de Dom Juan et qui représente une scène que les amateurs de théâtre attendent avec impatience, se trouve ici répercutée par l'image de Molière lui-même descendant dans la tombe au milieu des flammes, et constitue un indicateur non verbal de cette relation intertextuelle. Il n'est dès lors plus étonnant de constater que la pierre tombale porte le chiffre 1673, l'année de la mort de Molière.

Les images visuelles d'un feu ardent, qui comprennent les pages brûlées et les chaises enflammées, produisent un autre ensemble de relations intertextuelles, formé de citations du Doctor Faustus de Marlowe (section 9), de Lucrèce (section 5) et de Grimarest (section 5). Ce dernier met tous ces intertextes en contexte en révélant que Molière, qui avait traduit "presque tout Lucrèce», entra un jour dans une rage telle de voir l'un de ses serviteurs se servir des pages de sa traduction «pour en faire des papillottes» que «dans sa colère, il jeta sur-le-champ le reste au feu» (section 5). Jan Linders mentionne même que Molière brûla ses manuscrits et qu'ainsi «aucun d'eux ne nous est parvenu ${ }^{6}$. On doit aussi faire remarquer que le De rerum natura de Lucrèce est un poème didactique et lyrique qui expose la doctrine épicurienne, et que le Docteur Faustus brûla ses livres en concluant un pacte avec Lucifer (V.2) ${ }^{7}$. Il semble bien qu'ainsi l'intention de Wilson et Müller ait été de projeter une impression d'énergie infernale sur l'image de Molière, en accord avec celle que véhicule Dom Juan.

Dom Juan combine ses inclinations hédonistes et hérétiques avec, au début, une idéologie humaniste qui sera plus tard remplacée par une pure hypocrisie religieuse. De plus, son apparent humanisme semble se fonder sur une sorte d'attitude scientifique à l'endroit de l'univers. Quand Sganarelle le questionne sur ses croyances, il répond:

Dom Juan: Je crois que deux et deux sont quatre, Sganarelle, et que quatre et quatre sont huit.

Sganarelle: La belle croyance que voilà! Votre religion [...] est donc l'arithmétique? (III, 1)

Le rattachement de l'humanisme scientifique moderne à la figure du «méchant homme» indique clairement une critique implicite, comme chaque fois qu'une attitude est liée à un personnage ridicule. De plus, vers la fin de la pièce, Molière change les caractéristiques de Dom Juan et ajoute à sa nature libertine l'hypocrisie religieuse comme le meilleur moyen de manifester son vil hédonisme ( IIl n'est rien de tel en ce monde que de se contenter", I, 2): 
Sganarelle: Quoi! Vous ne croyez rien du tout, et vous voulez cependant vous ériger en homme de bien?

Dom Juan: Et pourquoi non? Il y en a tant d'autres comme moi, qui se mêlent de ce métier et qui se servent du même masque pour abuser le monde! (V, 2)

En fait, Dom Juan exploite alternativement la science et la religion à ses propres fins condamnables et, on le sait, le faux-médecin et le faux-dévot représentent justement les cibles favorites de Molière.

L'image visuelle de Colbert donnant une pièce de monnaie à Molière (section 2), venant illustrer la formule «Molière c'est la PÉNURIE» (section 1), est liée intertextuellement à l'épisode de Dom Juan dans lequel l'anti-chevalier qu'est Dom Juan promet de donner un louis d'or 8 à un pauvre à condition que celui-ci blasphème. Ceci rappelle la rente que reçut Molière du roi pour le dédommager de l'interdiction de ses deux pièces, Le Tartuffe et Dom Juan, et ne le montre pas tout à fait sous les traits d'un champion de la morale, mais plutôt comme un serviteur au service du roi dans son conflit avec le clergé. Quoi qu'il en soit, l'attaque lancée par Molière contre les faux-dévots eut un effet qui vient renforcer la formule du texte «Molière c'est une danse / Sur une petite scène parmi les poignards affûtés du clergé» (section 1).

Tous ces motifs indiquent clairement que Wilson et Müller attribuent à Molière certains traits de son propre Dom Juan: le critique moral se fait à son tour juger selon ses propres critères. Son image s'en trouve déconstruite. Et à partir des mêmes critères, Wilson et Müller s'attribuent à eux-mêmes certains traits de Molière.

\section{LES FEMMES SAVANTES}

Les images de Molière sur son lit de mort, criant comme un animal et aboyant comme un chien ${ }^{9}$, conjuguées au texte de Müller qui prétend que l'humanité représente «une expérience / Entre ange et bête" (section 1), entretiennent des relations intertextuelles avec le passage des Femmes savantes où Armande oppose la rationalité de l'homme à sa tendance à vouloir satisfaire ses instincts les plus bas et même des instincts animaux:
Armande: Mariez-vous, ma sœur, à la philosophie, Qui nous monte au-dessus de tout le genre humain, Et donne à la raison l'empire souverain, Soumettant à ses lois la partie animale, Dont l'appétit grossier aux bêtes nous ravale. $(\mathrm{I}, 1)^{10}$

La tentative générale de déconstruction de l'image de Molière acquiert là une dimension supplémentaire. Celui-ci doit être ainsi conçu comme une combinaison d'un génie de nature divine et d'une énergie animale, semblable à l'énergie infernale manifestée par Dom Juan. En ce sens, l'image visuelle d'un renard sorti tout droit de La Fontaine et prenant place sur scène constitue une bonne métaphore de Molière. Dans la fable, les animaux ne se contentent pas de présenter des traits humains: leurs caractéristiques animales forment le prédicat métaphorique et leur comportement semblable à celui des humains renvoie au référent de cette métaphore.

Semblablement, cette façon dualiste de concevoir l'humanité présuppose une approche religieuse et même chrétienne, approche dont la rigueur ne correspond guère à la sensibilité moderne et postmoderne. Mais l'hédonisme bestial, l'adultère, l'hypocrisie et même l'hérésie - au sens large - demeurent des tabous universels de l'humanité. La religion voit en l'homme un ange, alors que la science considère qu'il fait fondamentalement partie de l'espèce animale. Il n'y a donc aucune chance que l'une ou l'autre comprenne la nature humaine.

\section{QUESTION DE STRUCTURE}

Les relations intertextuelles entre les pièces de Molière et son bio-texte permettent une nouvelle interprétation de ses personnages principaux, vus comme des personnifications ou plutôt des projections métaphoriques de sa propre nature qui forment ainsi sa propre autobiographie. En ce sens, la citation que fait Müller du As You Like It de Shakespeare ${ }^{11}$ ("le monde entier est un théâtre») devient la clé du thème central de l'interpénétration des mondes réel et fictif. Dans cette perspective nouvelle, les personnages dont Molière se sert apparemment pour dénoncer les travers moraux de la société deviennent un commentaire ironique sur lui- 
même. Aux yeux de Wilson et de Müller, ses personnages, avec leurs défauts, reflètent mieux sa propre nature que ses prétentions bien payées. Ils déconstruisent en fait l'image du dramaturge telle que nous la donne son bio-texte ${ }^{12}$.

Cette relation métaphorique entre les personnages des comédies de Molière (prédicats) et l'auteur luimême dans son bio-texte (sujet/référent) se trouve redoublée dans la relation métaphorique entre Molière en tant que personnage de la vidéo (prédicat) et Wilson/Müller (sujet/référent). Wilson s'est fait le référent de la métaphore structurale en créant le personnage de fiction qu'est Molière et en jouant son rôle, comme si c'était le vrai Molière, mais en tenant compte de ses personnages de fiction. Quant à Müller, il s'est fait lui aussi le référent de la métaphore structurale en lisant le texte de la voix off, qui inclut également des intertitres et des citations tirées de son propre «Paysage avec Argonautes» ${ }^{13}$. On peut voir ce poème comme une prémonition de sa propre mort qui, ironiquement (comme la mort de Molière), est survenue dix jours après la première allemande de $L a$ Mort de Molière.

Cette double métaphore structurelle de la vidéo vient appuyer la formule liminaire «Ce n'est pas un poème sur Molière" (section 1). De fait, c'est un poème sur Wilson/Müller: un prédicat métaphorique qui semble à première vue renvoyer à deux référents, mais n'en admet finalement qu'un seul. La même chose s'applique à cette double structure métaphorique. La Mort de Molière représente une projection/personnification des angoisses de Wilson/ Müller. La déconstruction à l'œuvre au niveau du prédicat métaphorique (Molière) s'applique ainsi en dernière instance à eux-mêmes.

L'ironie est le gestus fondamental de cette ouvre. Cette ironie s'exerce sur le bio-texte de Molière, en particulier sur la gloire que lui a donnée la nation: «Molière est mort une motte de nature une pièce de la France» (section 10). La vidéo semble dire que la gloire posthume n'est qu'une construction culturelle et que la gloire nationale n'est qu'une autoglorification nationale. La nation ne fait ainsi que se célébrer elle-même. La nation est fière d'être une nation et n'éprouve au fond qu'indifférence pour la mort trop humaine de Molière. C'est sous le même angle que Wilson/Müller exercent leur auto-ironie sur leur propre valeur culturelle.

Cette vidéo constitue une métaphore extrêmement complexe et forme un réseau d'intertextes qui, à mon sens, s'ordonnent selon une perspective unique. Il présuppose un lecteur implicite proprement impossible: le texte est en effet bien plus complexe que ce que le spectateur ordinaire est capable de décoder. Il est en outre bien difficile de supposer que la déconstruction de l'image d'un héros culturel puisse avoir pour le spectateur le sens d'une invite à reconsidérer sa propre image. Mais il reste que ce spectateur est impliqué par la remise en question des images des héros culturels qu'il chérit, images que les promoteurs de la culture lui ont mises dans la tête. Ici la déconstruction tend à faire perdre à ces images leur familiarité, au nom de la vérité.

\footnotetext{
Notes

1. Robert Wilson et Heiner Müller: La Mort de Molière. Toutes les citations renvoient aux textes écrits et choisis par H. Müller, à partir de la version allemande de la bande image.

2. Molière, Le Malade imaginaire, Paris, Garnier-Flammarion, 1965,

vol. IV. Toutes les citations renvoient à cette édition du texte.

3. En français dans le texte (N.D.T.).

4. Molière, Dom Juan, Paris, Garnier-Flammarion, 1965, vol. II. Toutes

les citations renvoient à cette édition du texte.

5. Officiellement, Armande était la jeune sœur de son amante précédente, Madeleine Béjart, mais en coulisse on la pensait sa fille.

Son âge en tout cas concordait avec la période couverte par la relation amoureuse de Molière et de sa mère.

6. Jan Linders, "Molière \pm Müller ", Performance Research, vol. 1, n², 1996, p. 93-95.

7. Christopher Marlowe, Doctor Faustus, dans Eric Bentley, Five Plays, New York, Hill and Wang, 1956.

8. En français dans le texte (N.D.T.).

9. Sganarelle prétend que Dom Juan est un chien (Dom Juan, I, 1)

10. Molière, Les Femmes savantes, Paris, Garnier-Flammarion, 1965, vol. II.

11. «Le monde est un théâtre/ et tous hommes et toutes femmes ne sont que des acteurs; / Ils ont leurs sorties et leurs entrées / et un seul homme en son temps y tient plusieurs rôles ". Shakespeare, Comme il vous plaira, II, 6, p. 139-142 (cité dans la troisième section).

12. Cette déconstruction rappelle la déconstruction de l'image de l'artiste/écrivain dans La Mouette de Tchekhov.

13. Heiner Müller, "Paysage avec Argonautes », dans Germania Mort à Berlin, Paris, Éd. de Minuit, 1990.
} 